\title{
Evaluation of the Expression of Circulating miR-16 and miR-26a in the plasma of Gastric Cancer Patients in Guilan province, North of Iran
}

\author{
Tolou Babaei Hemmaty ${ }^{1}$, Najmeh Ranji $1^{1,}$ and Fatemeh Safari ${ }^{2}$ \\ 1 Department of Biology, Faculty of Sciences, Rasht Branch, Islamic Azad University, Rasht, Iran \\ 2 Department of Biology, Faculty of Sciences, University of Guilan, Rasht, Iran
}

* Corresponding author: Najmeh Ranji, Department of Biology, Faculty of Sciences, Rasht Branch, Islamic Azad University, Rasht, Iran. Tel: +981333424080; Email: n_ranji@iaurasht.ac.ir

Received 2020 December 06; Revised 2021 February 01; Accepted 2021 February 24.

\begin{abstract}
Background: Gastric cancer (GC) is a global health problem and the second deadly type of cancer worldwide with 1000 deaths per year. Poor prognosis in the early stages is one of the burdens in the treatment of GC. MicroRNAs are 18-22 nucleotide non-coding RNAs which play critical roles in the regulation of gene expression. Nowadays, miRNAs are widely known as non-invasive biomarkers for various kinds of cancers.

Objectives: This study aimed to evaluate the expression level of circulating miR-16 and miR-26a in GC patients and investigate the potential prognostic role of these miRNAs.

Methods: Initially, 20 plasma samples were obtained from pre-and post-operative GC patients, and the expression of miR-16 and -26a were compared with that of 20 healthy controls. The miRNAs expression was investigated using Real-Time quantitative PCR. The association between the expression levels of these miRNAs and clinicopathological features was also investigated in this study.

Results: MiR-16 was down-regulated in GC patients; however, miR-26a expression revealed no significant difference between patients and controls in this regard. Furthermore, the expression of two miRNAs showed no association with the grade, TNM stage, and smoking status of the patients. Eventually, decreased expression of miR-16 was not correlated with the expression level of miR-26a.

Conclusion: The downregulation of circulating miR-16 introduces this microRNA as a candidate biomarker for the non-invasive early prognosis of GC.

Keywords: Gastric cancer, miR-16, miR-26a, Plasma, Real-time quantitative PCR
\end{abstract}

\section{Background}

Gastric cancer (GC) is the second deadly cancer and the fourth most common type of cancer worldwide $(1,2)$. The early diagnosis of this disease is difficult due to the lack of sufficient typical GC symptoms which leads to hazardous implications in the advanced stages (3). It is worth mentioning that the process of GC pathogenesis is very complicated and involves many genetic and environmental factors (4). The main problems include poor prognosis and chemotherapy resistance which explain the need for developing novel biomarkers for the diagnosis and understanding the complete mechanism of GC tumorigenesis $(1,5)$.

In the last decade, microRNAs (miRNAs) have attracted much attention and led to the development of new treatment and diagnosis strategies (6). MiRNAs are a group of non-coding single-stranded RNAs with 17 to 25 nucleotides in length (7) which negatively regulate gene expression by targeting the complementary sequences of mRNAs (8). Today, several biomarkers are established based on circulating miRNAs for the diagnosis and prognosis of different cancers, such as lung, colorectal, ovarian, and pancreatic cancer (9). Moreover, many regulatory roles have been discussed for miRNAs in modulating gene expression levels, which can affect such processes as apoptosis, cell proliferation, and differentiation $(10,11)$. In a well-known mechanism, they bind to the miR response element in the 3'-untranslated region (3'-UTR) on mRNA, and this promotes its degradation or translational suppression (12). Moreover, they also upregulate some proteins in quiescent cells, such as oocytes, or upregulate proteins by binding to 5'-UTR in some specific conditions (13). Considering the vital regulatory roles of miRNAs, they can disrupt the cell cycle and impact the carcinogenesis processes. Numerous studies have shown that more than half of human miRNAs are associated with different types of cancer (14).

In the same vein, dysregulation of some miRNAs has oncogenic or tumor-suppressive impacts on GC carcinogenesis (15). In this regard, the overexpression of oncomiRs and downregulation of tumor-suppressor miRs are involved in cancer development (16).

MiR-16 and miR-26a are located in chromosomes $13 q 14$ and $3 p 21$, respectively (17-19). Both of these microRNAs have been reported to be involved in various types of cancers in many previous studies. In this regard, one can refer to the tumor-suppressing function of miR-16 in glioblastoma by targeting Wip1 (20). In a recent study, miR-16 was found to act as a tumor suppressor in GC through the suppression of 
SALL4, which is a zinc finger transcription factor, or through targeting SMAD3, which is a regulator of the TGF $\beta$ pathway (21). In many studies, miR-26a has also been found to be associated with carcinogenesis in a variety of tumors, such as gastric tumors. In other studies, miR-26a was defined as a tumor-suppressor miRNA that targets FGF9, NRAS, E2F2, and HGF (22-24).

In this study, the expression levels of miR-16 and miR-26a were evaluated in the plasma samples of the patients with GC from Guilan province, Iran, and compared with those in healthy individuals. The control samples were obtained from healthy people with no clinical or laboratory sign of gastrointestinal implications. Eventually, all patients were allowed to leave the study at any time, and the healthy cases included volunteers with no history of gastrointestinal diseases.

\section{Objectives}

This study aimed to evaluate the expression level of circulating miR-16 and miR-26a in GC patients and investigate the potential prognostic role of these miRNAs.

\section{Methods}

\subsection{Plasma samples}

The plasma samples (1-1.5 ml) were collected from pre- and post-operative GC patients who had not undergone therapies, such as radiotherapy or chemotherapy. The study samples were collected from the consenting individuals from Jam pathobiology laboratory and Razi Hospital, Rasht, Iran, between September 2018 and January 2020 and stored at $-70{ }^{\circ} \mathrm{C}$. Each GC patient was staged according to the American Joint Committee Cancer staging manual (25).

\subsection{Total RNA Extraction}

Plasma samples were incubated at room temperature, and subsequently, the total RNA of plasma was isolated using an YTzol Pure RNA kit (Yekta Tajhiz azma Co., Tehran, Iran). For this purpose, the total RNA content of plasma was isolated through precipitation, and the extracted RNA was resolved in RNase-free water and stored at -70 ${ }^{\circ} \mathrm{C}$. Concentration and purity of extracted RNA were assessed using NanoDrop 1000 (Thermo Scientific, Wilmington, DE) and absorbance value of 260/280.

\subsection{Real-time $P C R$}

The cDNA synthesis was accomplished using a BON-miR 1st-Strand cDNA synthesis kit (BON-miR, Tehran, Iran). In this case, the universal reverse ATP primer was used for poly (A) polymerization, and the BON-RT adaptor primer was used to synthesize total cDNA. The cDNA synthesis was followed by a qRT-
PCR reaction using BON-miR High-Specificity miRNA QPCR (BON-miR, Tehran, Iran) and RealQ Plus 2x Master Mix Green kits (Ampliqon, Odense, Denmark). Furthermore, the miR-93-5p was chosen as the internal control, and a universal reverse primer along with a specific forward primer was utilized to amplify the gene of interest. All reactions were carried out in triplicate using ABI StepOnePlus ${ }^{\mathrm{TM}}$ (ABI, CA, USA), and the raw data were primarily assessed and classified using ABI software. Accordingly, the delta $\mathrm{Ct}$ and $2^{-\Delta \Delta \mathrm{Ct}}$ methods were used to evaluate the expression level and compare the expression of miRNAs between patients and control groups, respectively.

\subsection{Statistical analysis}

All data collected from real-time PCR output were classified and analyzed using SPSS (version 21) (IBM Corp, N.Y., USA) and GraphPad Prism software (version 6) (GraphPad, CA, USA). P-values less than 0.05 were considered statistically significant. The Mann-Whitney and Spearman correlation coefficient were used to compare two unpaired groups without normal distribution and assess the correlation between different groups without normal distribution, respectively. Furthermore, the correlation between miRNA expression change and clinicopathological parameters was evaluated using Mann-Whitney and Kruskal-Wallis tests, respectively.

\section{Results}

\subsection{Basic information of patients}

The plasma samples were collected from pre- and post-operative GC patients with a mean age of 60 years. More than $80 \%$ of the patients had lymph node metastasis. Furthermore, more than half of the gastric tumors include cardia cancers, and the rest of them were antrum and body cancers. An experienced pathologist classified all the gathered demographic characteristics in this study (Table 1).

\begin{tabular}{lcc}
\hline \multicolumn{2}{l}{ Table 1. Pathological summary of the patients } \\
\hline Age & $>50$ & $14(70 \%)$ \\
& $<50$ & $6(30 \%)$ \\
Gender & Male & $12(60 \%)$ \\
& Female & $8(40 \%)$ \\
Smoking & Yes & $9(45 \%)$ \\
& No & $11(55 \%)$ \\
Lauren classification & Intestinal & $12(60 \%)$ \\
& Diffuse & $8(40 \%)$ \\
Tumor size & $<4 \mathrm{~cm}$ & $5(25 \%)$ \\
& $4-8 \mathrm{~cm}$ & $15(75 \%)$ \\
Tumour, node, & I & $3(15 \%)$ \\
metastasis stage & II & $4(20 \%)$ \\
& III & $12(60 \%)$ \\
& IV & $1(5 \%)$ \\
\multirow{2}{*}{ Differentiation grade } & Well & $4(20 \%)$ \\
& Moderate & $4(20 \%)$ \\
& Poor & $11(55 \%)$ \\
Depth of invasion & Undifferentiated & $1(5 \%)$ \\
& Serosal & $12(60 \%)$ \\
\hline
\end{tabular}



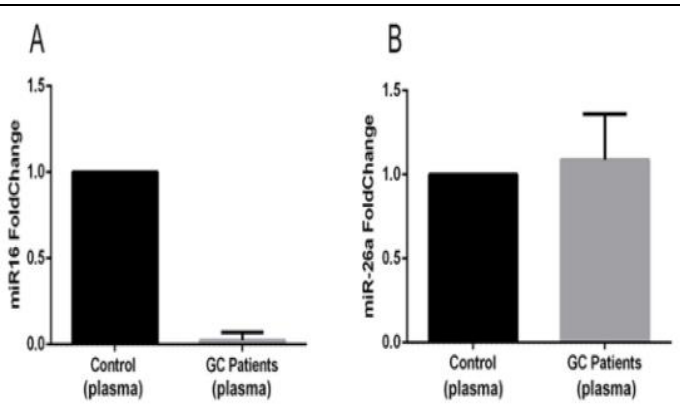

Figure 1. Logarithm base 10 for the fold change of the samples. The data under and above the zero line show downregulation and upregulation of the related sample, respectively. Black and gray bars represent miR-16 and miR-26a, respectively. This bar graph presents a summary of total gene expression changes among the patients. The mean FCs for miR-16 and miR-26a are 0.023 and 1.087 respectively.

\subsection{Expression of miR-16 and $\mathrm{miR}-26 a$}

The Ct values of the samples were classified, and the expression levels were studied using $2^{-\Delta \Delta \mathrm{Ct}}$ (fold change) values. The log fold change (FC) can effectively show which samples were overexpressed and which were underexpressed for the gene of interest. The logarithm base 10 of FC was used to present miRNAs expression changes in plasma samples of the patients (Figure 1).
All melting curves verified the specificity of PCR results. Following the primary classification of the qRT-PCR data, the FC values of miR-16 and -26a were compared through the Mann-Whitney test (Figure 2). Furthermore, the results revealed no significant difference between GC patients and the control group regarding the expression level of circulating miR-26a $(\mathrm{P}=0.0837)$; however, it showed a highly significant decrease in the expression level of miR-16 in plasma samples of GC patients, compared to that in the control group $(\mathrm{P}<0.0001)$.

\subsection{Correlation study between miR-16 and miR-26a}

The correlation between miR-16a and miR-26a was studied using Spearman's two-tailed test to find out whether their expression affected one another or not. There was no significant correlation between the expression of miR-16 and miR-26a among the patients $(\mathrm{P}=0.1986)$.

\subsection{Assessment of the correlation between the expression of miRNAs and clinicopathological characteristics}

The correlation between miRNAs expression and clinicopathological features was assessed using Mann-Whitney and Kruskal-Wallis tests. The expression level of both miR-16 and -26a were not

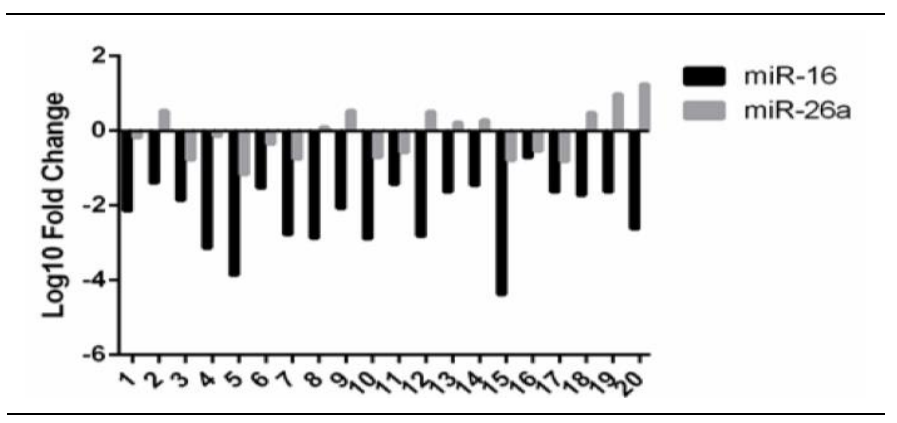

Figure 2. Comparison of fold change values between plasma samples of patients and control group. A) The fold change value of miR-16 was significantly lower in patients $(\mathrm{P}<0.0001)$. B) There was no significant difference between patients and the control group regarding the fold change value of miR-26a

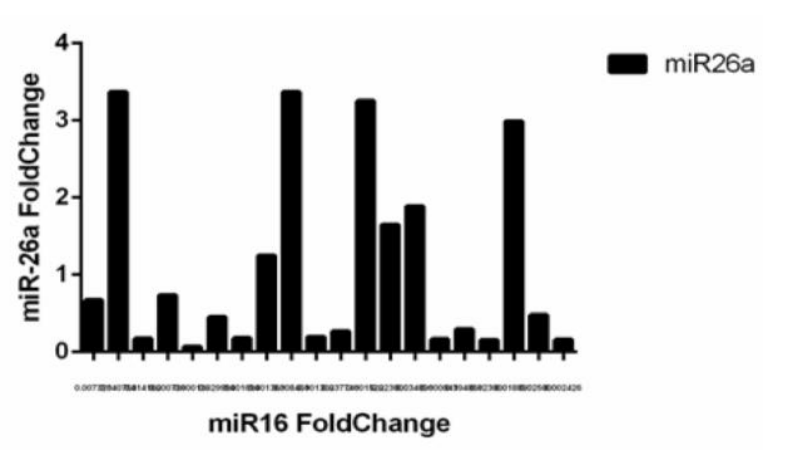

Figure 3. Assessment of correlation between miR16a and miR26a did not show a significant difference in plasma samples of the patients. The FCs of miR-26a and miR-16 are presented in Y and X axes, respectively. 
significantly associated with smoking; tumor, node, metastases stage; and tumor grade in plasma samples of the patients.

\section{Discussion}

The GC is one of the deadliest diseases worldwide, and it is regarded as the most common digestive system cancer [26]. Moreover, the study of the relationship between the expression of microRNAs and carcinogenesis of GC has been a challenge for researchers so far $(27,28)$. Currently, miRNA-based methods are great candidates for the development of new generations of biomarkers and target therapies for immune diseases and cancers (29). In this study, the expression levels of miR-16 and miR-26a were investigated in 20 plasma samples of GC patients and compared to those in healthy individuals.

The results of the present study revealed a significant decrease in the expression of miR-16a in the plasma of GC patients which was in line with the findings of former studies $(2,3,21,30)$. Despite the fact that miR-26a had a higher mean of FC, this difference was not significant. Recently, Yiran et.al reported the inhibitory roles of miR26a in carcinogenesis and angiogenesis of GC, which was in conflict with the findings of the present study. They found that miR26a/b binds to 3'-UTR of HGF mRNA and effectively downregulates the HGF expression. Therefore, the downregulation of miR26a could be accompanied by an increased rate of carcinogenesis in GC (23). In another study, Li et al. indicated the inhibitory roles of miR-26a in GC through downregulation of COL10A1, which is a principal part of the extracellular matrix and highly expressed in GC (31). Regarding the finding of the previous studies, the role of miR-26a in GC cannot be ruled out, and further studies on larger populations could contribute to the achievement of more reliable results.

Regarding the limitations of this study, one can refer to the low number of samples which can lead to insignificant differences between patients and controls.

This study particularly focused on miR-16 the downregulation of which was statistically significant. In line with the results of the present study, Chenyu et al. characterized the role of miR-16 downregulation in carcinogenesis of GC. They were attempting to find the mechanism involved in the anti-tumor effects of melatonin on GC cells using miRNA microarrays, which led to the finding of miR16-5p. They found that melatonin enhanced miR-16-5p expression and directly targeted and downregulated Smad3 (2). MicroRNA-16-5p, also known as a common internal reference, showed a certain level of expression in most of the tissues, plasma, and serum (32). In many studies, miR-16-5p has been downregulated in a different types of cancers (33). Zhang et al. studied the expression of dozens of miRNAs using genome-wide microarray profiling in plasma samples of GC patients, and only miR-16-5p and miR-19b-3p were downregulated among them (3).

It is worth mentioning that the oncogenic function of Smad3 has been reported in human cancers. The overexpression of Smad3 was found in human hepatocellular carcinoma and cervical cancer and was related to poor prognosis and higher progression $(34,35)$. MiR-16 also functions as a tumor suppressor by targeting Wip1 and SALL4 in many cancers, including GC. The Wip1 and SALL4 are serine/ threonine phosphatase and zinc finger transcription factors, respectively, and both are overexpressed in human cancers. It was found that Wip1 and SALL4 were negatively correlated with miR-16 and overexpressed by inhibiting miR-16 $(20,21,36)$. Moreover, the downregulation of miR16-1-3p directly enhanced the expression of Twist 1 and $B C L 2$ in GC (30). It should be noted that Bcl2 is one of the most studied oncogenes, and downregulation of miR16 has been associated with the de-repression of this gene in chronic lymphocytic leukemia (37).

These studies provide reliable evidence that was in line with the results of the present study and showed a highly significant decrease in miR-16 among GC patients. These results suggest that the circulating miR-16 could function as a tumor suppressor gene in GC patients and is directly associated with cancer progression and apoptosis inhibition.

Altogether, these findings pave the way for future studies to investigate the circulating miR-16 as a new non-invasive biomarker for the early diagnosis of GC.

\section{Conclusion}

In conclusion, while there was no significant difference between patients and healthy cases regarding the expression level of miR-26a, miR-16 could be a potential biomarker for GC since it showed a strongly significant decrease in patients $(P<0.0001)$. Eventually, a comparative study in which samples are collected before and after the gastrectomy surgery can effectively assess the ability of miR-16 as a new biomarker candidate for GC.

\section{Acknowledgments}

We would like to thank Hamid Saeidi Saedi for providing patient samples and information.

\section{Footnotes}

Authors' Contribution: T. B. H., and N. R. contributed for the conception and design of this article. T. B. H., and N. R. contributed for analysis and interpretation. T. B. H., N. R. and F. S. were involved in 
statistical analysis. T. B. H., and N. R. contributed in writing and provided critical revision for this article. N. R. gave final approval for this article. All authors agree to be accountable for all aspects of the work.

Conflict of Interests: The authors declared that no conflict of interest exists regarding this study.

Ethical Approval: This study was carried out in strict accordance with the recommendations of the ethics committee, and the study protocol was approved by the Ethics Committee of Human Experiments of Rasht Branch, Islamic Azad University, Rasht, Iran (IR.IAU.RASHT.REC.1399.027).

Funding/Support: This study has been funded by Islamic Azad University, Rasht, Iran (17/16/ 4/16035).

\section{References}

1. Hu ML, Xiong SW, Zhu SX, Xue XX, Zhou XD. MicroRNAs in gastric cancer: from bench to bedside. Neoplasma. 2019; 66(2):176-86. doi: 10.4149/neo_2018_180703N439. [PubMed: 30509106].

2. Zhu C, Huang Q, Zhu H. Melatonin inhibits the proliferation of gastric cancer cells through regulating the mir-16-5p-smad3 pathway. DNA Cell Biol. 2018;37(3): 244-52. doi: 10.1089/ dna.2017.4040. [PubMed: 29359963].

3. Zhang J, Song Y, Zhang C, Zhi X, Fu H, Ma Y, et al. Circulating MiR-16-5p and MiR-19b-3p as Two Novel Potential Biomarkers to Indicate Progression of Gastric Cancer. Theranostics. 2015;5(7):733-45. doi: 10.7150/thno.10305. [PubMed: 25897338].

4. Digklia A. , Wagner AD. Advanced gastric cancer: Current treatment landscape and future perspectives. World $J$ Gastroenterol. 2016;22(8):2403-14. doi: 10.3748/wjg.v22.i8.2403. [PubMed: 26937129].

5. Yang SM, Huang C, Li XF, Yu MZ, He Y, Li J. miR-21 confers cisplatin resistance in gastric cancer cells by regulating PTEN. Toxicology. 2013; 306:162-8. doi: 10.1016/j.tox.2013.02.014. [PubMed: 23466500].

6. Zhao X, Hu GF, Shi YF, Xu W. Research Progress in microRNABased Therapy for Gastric Cancer. Onco Targets Ther. 2019; 12:11393-411.

7. Tehrani FK, Ranji N, Kouhkan F, Hosseinzadeh S. Apoptosis induction and proliferation inhibition by silibinin encapsulated in nanoparticles in MIA PaCa-2 cancer cells and deregulation of some miRNAs. Iran J Basic Med Sci. 2020;23(4):469-82. doi: 10.22038/ijbms.2020.39427.9349. [PubMed: 32489562].

8. Zadeh MM, Motamed N, Ranji N, Majidi M, Falahi F. Silibinininduced apoptosis and downregulation of microrna-21 and microrna-155 in mcf-7 human breast cancer cells. J Breast Cancer;2016;19(1):45-52. doi: 10.4048/jbc.2016.19.1.45. [PubMed: 27066095].

9. Wang H, Peng R, Wang J, Qin Z, Xue L. Circulating microRNAs as potential cancer biomarkers: the advantage and disadvantage. Clin Epigenetics. 2018;10:59. doi: 10.1186/s13148-018-0492-1. [PubMed: 29713393].

10. Jamali L, Tofigh R, Tutunchi S, Panahi G, Borhani F, Akhavan S. Circulating microRNAs as diagnostic and therapeutic biomarkers in gastric and esophageal cancers. J Cell Physiol. 2018;233(11):8538-50. doi: 10.1002/jcp.26850. [PubMed: 29923196].

11. Ranji N, Sadeghizadeh M, Shokrgozar MA, Bakhshandeh B, Karimipour M, Amanzadeh A, et al. MiR-17-92 cluster: an apoptosis inducer or proliferation enhancer. Mol Cell Biochem. 2013;380(1-2):229-38. doi: 10.1007/s11010-013-1678-7. [PubMed: 23681423].

12. Pakizehkar S, Ranji N, Naderi Sohi A, Sadeghizadeh M. Curcumin loaded PEG400-OA nanoparticles: A suitable system to increase apoptosis, decrease migration, and deregulate
miR-125b/miR182 in MDA-MB-231 human breast cancer cells. Polymers Advanced Technol. 2020;31(8):1793-804. doi: 10.1002/pat.4906

13. O'Brien J, Hayder H, Zayed Y, Peng C. Overview of MicroRNA Biogenesis, Mechanisms of Actions, and Circulation. Front Endocrinol (Lausanne). 2018;9:402. doi: 10.3389/fendo. 2018.00402. [PubMed: 30123182].

14. Tsai MM, Wang CS, Tsai CY, Huang HW, Chi HC, Lin YH, et al. Potential Diagnostic, Prognostic and Therapeutic Targets of MicroRNAs in Human Gastric Cancer. Int J Mol Sci. 2016; 17(6):945. doi: 10.3390/ijms17060945. [PubMed: 27322246].

15. Chen B, Guo S, Yu Z, Feng Y, Hui L. Downregulation of microRNA-375, combined with upregulation of its target gene Janus kinase 2, predicts unfavorable prognosis in patients with gastric cancer. Int J Clin Exp Pathol. 2017;10(11):11106-13. [PubMed: 31966459].

16. Hossainzadeh S, Ranji N, Naderi Sohi A, Najafi F. Silibinin encapsulation in polymersome: A promising anticancer nanoparticle for inducing apoptosis and decreasing the expression level of miR-125b/miR-182 in human breast c ancer cells. J Cell Physiol. 2019;234(12):22285-98. doi: 10.1002/jcp.28795. [PubMed: 31073992].

17. Mohamed JS, Lopez MA, Boriek AM. Mechanical stretch upregulates microRNA-26a and induces human airway smooth muscle hypertrophy by suppressing glycogen synthase kinase-3beta. J Biol Chem. 2010;285(38):29336-47. doi: 10.1074/jbc.M110.101147. [PubMed: 20525681].

18. Li F, Xu Y, Deng S, Li Z, Zou D, Yi S, et al. MicroRNA-15a/16-1 cluster located at chromosome 13q14 is down-regulated but displays different expression pattern and prognostic significance in multiple myeloma. Oncotarget, 2015;6(35): 38270-82. doi: 10.18632/oncotarget.5681. [PubMed: 26516702].

19. Braga TV, Evangelista FC, Gomes LC, da Silva Araújo SS, das Graças Carvalho M, de Paula Sabino A. Evaluation of MiR-15a and MiR-16-1 as prognostic biomarkers in chronic lymphocytic leukemia. Biomed Pharmacother. 2017;92:864-9. doi: 10.1016/j.biopha.2017.05.144. [PubMed: 28599250].

20. Zhan XH, Xu QY, Tian R, Yan H, Zhang M, Wu J, et al. MicroRNA16 regulates glioma cell proliferation, apoptosis and invasion by targeting Wip1-ATM-p53 feedback loop. Oncotarget. 2017; 8(33):54788-98. doi: 10.18632/oncotarget.18510. [PubMed: 28903382].

21. Jiang X, Wang Z. miR-16 targets SALL4 to repress the proliferation and migration of gastric cancer. Oncol Lett. 2018;16(3):3005-12. doi: 10.3892/ol.2018.8997. [PubMed: 30127890].

22. Deng M, Tang HL, Lu XH, Liu MY, Lu XM, Gu YX, et al. miR-26a suppresses tumor growth and metastasis by targeting FGF9 in gastric cancer. PLoS One; 2013;8(8):e72662. doi: 10.1371/journal.pone.0072662. [PubMed: 24015269].

23. Si Y, Zhang H, Ning T, Bai $M$, Wang $Y$, Yang $H$, et al. miR-26a/b Inhibit Tumor Growth and Angiogenesis by Targeting the HGFVEGF Axis in Gastric Carcinoma. Cell Physiol Biochem. 2017;42(4):1670-83. doi: 10.1159/000479412. [PubMed: 28738343].

24. Wen L, Cheng F, Zhou Y, Yin C. MiR-26a enhances the sensitivity of gastric cancer cells to cisplatin by targeting NRAS and E2F2. Saudi J Gastroenterol. 2015;21(5):313-9. doi: 10.4103/1319-3767.166206. [PubMed: 26458859].

25. Edge SB, Compton CC. The American Joint Committee on Cancer: the 7th Edition of the AJCC Cancer Staging Manual and the Future of TNM. Ann Surg Oncol. 2010;17(6):1471-4. doi: 10.1245/s10434-010-0985-4. [PubMed: 20180029].

26. Siegel RL, Miller KD, Jemal A. Cancer Statistics, 2017. CA Cancer J Clin. 2017;67(1):7-30. doi: 10.3322/caac.21387. [PubMed: 28055103].

27. Liu L, Chen Q, Lai R, Wu X, Wu X, Liu F, et al. Elevated expression of mature miR-21 and miR-155 in cancerous gastric tissues from Chinese patients with gastric cancer. J Biomed Res, 2010. 24(3):187-97. doi: 10.1016/S1674-8301(10)60028-0. [PubMed: 23554630].

28. Link A, Kupcinskas J. MicroRNAs as non-invasive diagnostic biomarkers for gastric cancer: Current insights and future perspectives. World J Gastroenterol. 2018;24(30):3313-29. doi: 
10.3748/wjg.v24.i30.3313. [PubMed: 30122873].

29. Ranji N, Sadeghizadeh M, Karimipoor M, Shokrgozar MA, Sistani RN, Paylakhi SH. MicroRNAs Signature in IL-2-Induced CD4+ T Cells and Their Potential Targets. Biochem Genet. 2015;53(7-8): p. 169-83. doi: 10.1007/s10528-015-9677-x. [PubMed: 26048624].

30. Wang T, Hou J, Li Z, Zheng Z, Wei J, Song D, et al. miR-15a-3p and miR-16-1-3p Negatively Regulate Twist1 to Repress Gastric Cancer Cell Invasion and Metastasis. Int J Biol Sci. 2017;13(1):122-34. doi: 10.7150/ijbs.14770. [PubMed: 28123352].

31. Li HH, Wang JD, Wang W, Wang HF, Lv JQ.. Effect of miR-26a$5 \mathrm{p}$ on gastric cancer cell proliferation, migration and invasion by targeting COL10A1. Eur Rev Med Pharmacol Sci.2020; 24(3):1186-94. doi: 10.26355/eurrev_202002_20170. [PubMed: 32096148].

32. Reid G, Kirschner MB, van Zandwijk N. Circulating microRNAs: Association with disease and potential use as biomarkers. Crit Rev Oncol Hematol.2011;80(2):193-208. doi: 10.1016/j. critrevonc.2010.11.004. [PubMed: 21145252].

33. Ma Q, Wang X, Li Z, Li B, Ma F, Peng L, et al. microRNA-16 represses colorectal cancer cell growth in vitro by regulating the p53/survivin signaling pathway. Oncol Rep. 2013; 29(4):1652-8. doi: 10.3892/or.2013.2262. [PubMed: 23380758].

34. Zhou Q, Han LR, Zhou YX, Li Y. MiR-195 Suppresses Cervical Cancer Migration and Invasion Through Targeting Smad3. Int ] Gynecol Cancer. 2016;26(5):817-24. doi: 10.1097/IGC.000000 0000000686. [PubMed: 27206216].

35. Zhou HH, Chen L, Liang HF, Li GZ, Zhang BX, Chen XP. Smad3 Sensitizes Hepatocelluar Carcinoma Cells to Cisplatin by Repressing Phosphorylation of AKT. Int J Mol Sci. 2016; 17(4):610. doi: 10.3390/ijms17040610. [PubMed: 27110775].

36. Tatetsu H, Kong NR, Chong G, Amabile G, Tenen DG, Chai L. SALL4, the missing link between stem cells, development and cancer. Gene. 2016;584(2):111-9. doi: 10.1016/j.gene.2016.02.019. [PubMed: 26892498].

37. Lin K, Farahani M, Yang Y, Johnson GG, Oates M, Atherton M, et al. Loss of MIR15A and MIR16-1 at 13q14 is associated with increased TP53 mRNA, de-repression of BCL2 and adverse outcome in chronic lymphocytic leukaemia. $\mathrm{Br} J$ Haematol 2014;167(3):346-55. doi: 10.1111/bjh.13043. [PubMed: 25040181] 